\title{
Hierarchical Comprehensive Triangular Decomposition $^{\star}$
}

\author{
Zhenghong Chen, Xiaoxian Tang, and Bican Xia \\ School of Mathematical Sciences, Peking University, China \\ septemwnid@pku.edu.cn, tangxiaoxian@pku.edu.cn, xbc@math.pku.edu.cn
}

\begin{abstract}
The concept of comprehensive triangular decomposition (CTD) was first introduced by Chen et al. in their CASC'2007 paper and could be viewed as an analogue of comprehensive Gröbner systems for parametric polynomial systems. The first complete algorithm for computing CTD was also proposed in that paper and implemented in the RegularChains library in Maple. Following our previous work on generic regular decomposition for parametric polynomial systems, we introduce in this paper a so-called hierarchical strategy for computing CTDs. Roughly speaking, for a given parametric system, the parametric space is divided into several sub-spaces of different dimensions and we compute CTDs over those sub-spaces one by one. So, it is possible that, for some benchmarks, it is difficult to compute CTDs in reasonable time while this strategy can obtain some "partial" solutions over some parametric sub-spaces. The program based on this strategy has been tested on a number of benchmarks from the literature. Experimental results on these benchmarks with comparison to RegularChains are reported and may be valuable for developing more efficient triangularization tools.
\end{abstract}

Keywords: Comprehensive triangular decomposition, regular chain, hierarchical, generic regular decomposition, parametric polynomial system.

\section{Introduction}

Solving parametric polynomial system plays a key role in many areas such as automated geometry theorem deduction, stability analysis of dynamical systems, robotics and so on. For an arbitrary parametric system, in symbolic computation, solving this system is to convert equivalently the parametric system into new systems with special structures so that it is easier to analyze or solve the solutions to the new systems. There are two main kinds of symbolic methods to solve parametric systems, i.e., the algorithms based on Gröbner bases [12]14[15]16]17|23] and those based on triangular decompositions [12|5|9|11|13|19|20|24|25|26|27.

The methods based on triangular decompositions have been studied by many researchers since Wu's work [24] on characteristic sets. A significant concept in the theories of triangular sets is regular chain (or normal chain) introduced

\footnotetext{
* The work was supported by National Science Foundation of China (Grants 11290141 and 11271034).
} 
by Yang and Zhang 27] and Kalkbrener [11 independently. Gao and Chou proposed a method in [9] for identifying all parametric values for which a given system has solutions and giving the solutions by $p$-chains without a partition of the parameter space. Wang generalized the concept of regular chain to regular system and gave an efficient algorithm for computing it 20/21/22. It should be noticed that, due to their strong projection property, the regular systems or series may also be used as representations for parametric systems. Chen et. al. introduced the concept of comprehensive triangular decomposition (CTD) [5] to solve parametric systems, which could be viewed as an analogue of comprehensive Gröbner systems. Algorithm CTD for computing CTD was also proposed in $[5]$.

There are several implementations based on the above triangularization methods, such as Epsilon [22, RegularChains [6] and wsolve [19].

Suppose $\mathbf{P} \subset \mathbb{Q}[U][X]$ is a parametric polynomial system where $X=\left(x_{1}, \ldots\right.$, $\left.x_{n}\right)$ are variables and $U=\left(u_{1}, \ldots, u_{d}\right)$ are parameters. The above mentioned algorithms all solve the system in $\mathbb{C}^{d+n}$ directly. That means all the unknowns $(U$ and $X)$ are viewed as variables and triangular decompositions are computed over $\mathbb{Q}$. It may happen that no triangular decompositions over $\mathbb{Q}$ can be obtained in a reasonable time for some systems while a triangular decomposition over $\mathbb{Q}[U]$ is much easier to be computed.

Based on this observation, we propose a strategy which computes CTDs for given parametric systems hierarchically and the CTDs are called hierarchical comprehensive triangular decompositions (HCTD). By "hierarchical" we mean that, roughly speaking, a generic regular decomposition is computed first over $\mathbb{Q}[U]$ and a parametric polynomial $B(U)$ is obtained at the same time such that the solutions to the original system in $\mathbb{C}^{d+n}$ can be expressed as the union of solutions to those regular systems in the decomposition provided that the parameter values satisfying $B(U) \neq 0$. Then, by applying similar procedure recursively, the solutions satisfying $B(U)=0$ are obtained through adding $B(U)=0$ to the system and treating some parameters as variables. We give an algorithm based on this hierarchical strategy which computes CTDs for given parametric systems. The algorithm has been implemented with Maple and tested on a number of benchmarks from the literature. Experimental results on these benchmarks with comparison to RegularChains are reported (see Tables 21) and may be valuable for developing more efficient triangularization tools. For some benchmarks, it is difficult to compute CTDs in reasonable time while our program can output "partial solutions" (see Table 4).

The rest part of this extended abstract is organized as follows. Section 2 introduces an algorithm, Algorithm HCTD, for computing CTDs hierarchically and an example is illustrated there. Section 3 compares the Algorithm HCTD and the Algrotihm CTD in [5] by experiments. Section 4 introduces another hierarchical strategy for computing CTD and the comparing experiments are also shown. Section 5 shows the benefit of the hierarchical strategy by experiments. 


\section{Algorithm HCTD}

For the concepts and notations without definitions, please see [2]216].

Suppose $\mathbf{T}$ is a regular chain in $\mathbb{Q}[U][X]$ and $\mathbf{H} \subset \mathbb{Q}[U][X]$. [T, $\mathbf{H}]$ is said to be a regular system $[5$ if $\operatorname{res}(H, \mathbf{T}) \neq 0$ for any $H \in \mathbf{H}$. For any $\mathbf{B} \subset \mathbb{Q}[U]$, $\mathrm{V}^{U}(\mathbf{B})$ denotes the set $\left\{\left(a_{1}, \ldots, a_{d}\right) \in \mathbb{C}^{d} \mid B\left(a_{1}, \ldots, a_{d}\right)=0, \forall B \in \mathbf{B}\right\}$. For any $\mathbf{P} \subset \mathbb{C}[X], \mathrm{V}(\mathbf{P})$ denotes the set $\left\{\left(b_{1}, \ldots, b_{n}\right) \in \mathbb{C}^{n} \mid P\left(b_{1}, \ldots, b_{n}\right)=0, \forall P \in\right.$ $\mathbf{P}\}$. For any $\mathbf{P} \subset \mathbb{Q}[U][X], \mathrm{V}(\mathbf{P})$ denotes the set $\left\{\left(a_{1}, \ldots, a_{d}, b_{1}, \ldots, b_{n}\right) \in\right.$ $\left.\mathbb{C}^{d+n} \mid P\left(a_{1}, \ldots, a_{d}, b_{1}, \ldots, b_{n}\right)=0, \forall P \in \mathbf{P}\right\}$. For $D \subset \mathbb{C}^{d+n}$, denote by $\Pi_{U}(D)$ the set $\left\{\left(a_{1}, \ldots, a_{d}\right) \in \mathbb{C}^{d} \mid\left(a_{1}, \ldots, a_{d}, b_{1}, \ldots, b_{n}\right) \in D\right\}$. Suppose $[\mathbf{T}, \mathbf{H}]$ is a regular system in $\mathbb{Q}[U][X]$. If $\mathbf{H}=\{H\}$, then $[\mathbf{T}, \mathbf{H}]$ is denoted by $[\mathbf{T}, H]$ for short.

Due to page limitation, we only present the specification of an algorithm for computing CTDs hierarchically.

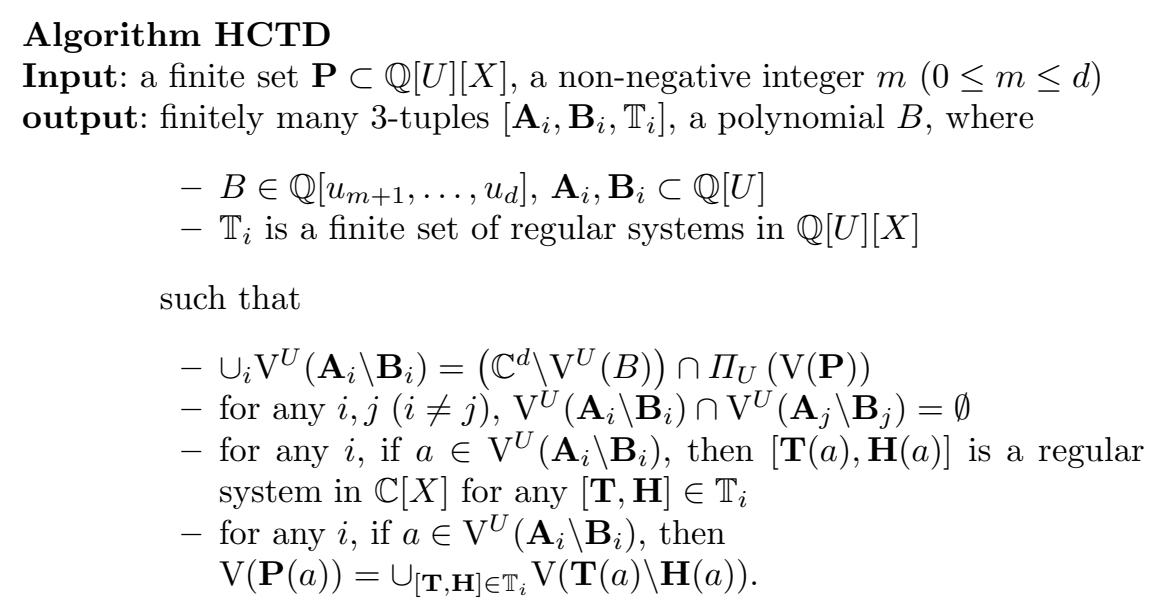

The output of $\operatorname{HCTD}(\mathbf{P}, m)$ is called the $m$-HCTD of $\mathbf{P}$. Each $\left[\mathbf{A}_{i}, \mathbf{B}_{i}, \mathbb{T}_{i}\right]$ in the $m$-HCTD is called a branch. Each regular system in the set $\cup \mathbb{T}_{i}$ is called a grape. By Algorithm HCTD, for any $\mathbf{P}$, if $m=0$, the output is the so-called generic regular decomposition [8] of $\mathbf{P}$; if $m=d$, the output is the comprehensive triangular decomposition [5] of $\mathbf{P}$. The Example 1 below shows how to get $m$ $\operatorname{HCTD}(m=0, \ldots, d)$.

Example 1 Consider the parametric system

$$
\mathbf{P}=\left\{\begin{array}{l}
2 x_{2}^{2}\left(x_{2}^{2}+x_{1}^{2}\right)+\left(u_{2}^{2}-3 u_{1}^{2}\right) x_{2}^{2}-2 u_{2} x_{2}^{2}\left(x_{2}+x_{1}\right)+2 u_{1}^{2} u_{2}\left(x_{2}+x_{1}\right) \\
-u_{1}^{2} x_{1}^{2}+u_{1}^{2}\left(u_{1}^{2}-u_{2}^{2}\right) \\
4 x_{2}^{3}+4 x_{2}\left(x_{2}^{2}+x_{1}^{2}\right)-2 u_{2} x_{2}^{2}-4 u_{2} x_{2}\left(x_{2}+x_{1}\right)+2\left(u_{2}^{2}-3 u_{1}^{2}\right) x_{2}+2 u_{1}^{2} u_{2}, \\
4 x_{1} x_{2}^{2}-2 u_{2} x_{2}^{2}-2 u_{1}^{2} x_{1}+2 u_{1}^{2} u_{2} .
\end{array}\right.
$$

where $x_{1}, x_{2}$ are variables and $u_{1}, u_{2}$ are parameters.

1. By the Algorithm RDU in [8], we compute a set $\mathbb{T}_{1}$ of regular systems and a polynomial $B_{1}\left(u_{1}, u_{2}\right)$ such that if $B_{1}\left(u_{1}, u_{2}\right) \neq 0$, then the solution set of $\mathbf{P}=0$ is equal to the union of the solution sets of the regular systems in $\mathbb{T}_{1}$. Then we obtain the 0-HCTD of $\mathbf{P}:\left[\mathbf{A}_{1}, \mathbf{B}_{1}, \mathbb{T}_{1}\right]$. 
2. Let $\mathbf{P}_{1}=\mathbf{P} \cup\left\{B_{1}\right\}$. Regard $\left\{u_{1}, x_{1}, x_{2}\right\}$ as the new variable set. By the Algorithm $\mathrm{RDU}$, we compute a set $\mathbb{S}_{1}$ of regular systems and a polynomial $B_{2}\left(u_{2}\right)$ such that if $B_{1}\left(u_{1}, u_{2}\right)=0$ and $B_{2}\left(u_{2}\right) \neq 0$, then the solution set of $\mathbf{P}=0$ is equal to the union of the solution sets of the regular systems in $\mathbb{S}_{1}$. For $\mathbb{S}_{1}$, applying the similar method as the Algorithm RegSer in [20] and the Algorithms Difference and CTD in [6], we obtain the 1-HCTD of $\mathbf{P}:\left[\mathbf{A}_{1}, \mathbf{B}_{1}, \mathbb{T}_{1}\right], \ldots,\left[\mathbf{A}_{4}, \mathbf{B}_{4}, \mathbb{T}_{4}\right]$.

3. Let $\mathbf{P}_{2}=\mathbf{P}_{1} \cup\left\{B_{2}\right\}$. Regard $\left\{u_{2}, u_{1}, x_{1}, x_{2}\right\}$ as the new variable set. By the Algorithm RDU, we compute a set of regular systems $\mathbb{S}_{2}$ and a polynomial $B_{3}=1$ such that if $B_{1}\left(u_{1}, u_{2}\right)=0, B_{2}\left(u_{2}\right)=0$ and $B_{3} \neq 0$, then the solution set of $\mathbf{P}=0$ is equal to the union of the solution sets of the regular systems in $\mathbb{S}_{2}$. For $\mathbb{S}_{2}$, applying the similar method as the Algorithms RegSer, Difference and CTD, we obtain the 2-HCTD of $\mathbf{P}:\left[\mathbf{A}_{1}, \mathbf{B}_{1}, \mathbb{T}_{1}\right], \ldots,\left[\mathbf{A}_{6}, \mathbf{B}_{6}, \mathbb{T}_{6}\right]$.

Table $1\left[\mathbf{A}_{i}, \mathbf{B}_{i}, \mathbb{T}_{i}\right]$

\begin{tabular}{c|c|c|c}
\hline$i$ & $\mathbf{A}_{i}$ & $\mathbf{B}_{i}$ & $\mathbb{T}_{i}$ \\
\hline 1 & $\emptyset$ & $\left\{u_{1} u_{2}\left(u_{1}^{2}-u_{2}^{2}\right)\right\}$ & $\left\{\left[\left\{-2 x_{1}^{2}+3 x_{1} u_{2}-u_{2}^{2}+u_{1}^{2}, 2 x_{1} x_{2}+u_{1}^{2}-u_{2} x_{2}\right\}, u_{1}\right]\right\}$ \\
\hline 2 & $\left\{u_{1}\right\}$ & $\left\{u_{2}\right\}$ & $\left\{\left[\left\{-2 x_{1}+u_{2}, u_{2}-2 x_{2}\right\}, 1\right]\right\}$ \\
\hline 3 & $\left\{u_{1}-u_{2}\right\}$ & $\left\{u_{2}\right\}$ & $\left\{\left[\left\{x_{1}, x_{2}-u_{2}\right\}, 1\right],\left[\left\{2 x_{1}-3 u_{2}, x_{2}+u_{2}\right\}, 1\right]\right\}$ \\
\hline 4 & $\left\{u_{1}+u_{2}\right\}$ & $\left\{u_{2}\right\}$ & $\left\{\left[\left\{x_{1}, x_{2}-u_{2}\right\}, 1\right],\left[\left\{2 x_{1}-3 u_{2}, 2 x_{2}+u_{2}\right\}, 1\right]\right\}$ \\
\hline 5 & $\left\{u_{2}\right\}$ & $\left\{u_{1}\right\}$ & $\left\{\left[\left\{2 x_{1}^{2}-u_{1}^{2}, 2 x_{2}^{2}-u_{1}^{2}\right\}, 1\right]\right\}$ \\
\hline 6 & $\left\{u_{1}, u_{2}\right\}$ & $\{1\}$ & $\left\{\left[\left\{x_{2}\right\}, 1\right],\left[\left\{x_{1}, x_{2}\right\}, 1\right],\left[\left\{2 x_{1}^{2}-u_{1}^{2}, 2 x_{2}^{2}-u_{1}^{2}\right\}, 1\right]\right\}$ \\
\hline
\end{tabular}

\section{Experiment of Comparison}

We have implemented the Algorithm HCTD as a Maple function HCTD and tested a great many benchmarks from the references 7 7/5]12[14. Throughout this paper, all the computational results are obtained in Maple 17 using an Intel(R) Core(TM) i5 processor (3.20 GHz CPU), 2.5 GB RAM and Windows 7 (32 bit). All the timings are given by seconds. The "timeout" mark means the time is greater than 1000 seconds. The Table 2 compares the functions HCTD (when $m=d)$ and ComprehensiveTriangularize (CTD) in RegularChains.

In Table 2, the column "time" lists the timings of HCTD $(m=d)$ and CTD; the column "branch" lists the numbers of branches output by HCTD and CTD; and the column "grape" lists the numbers of grapes output by HCTD and CTD. It is indicated by Table 2 that

- for the benchmarks 3-27, HCTD runs faster than CTD, especially, for the benchmark 27, CTD is timeout and HCTD completes the computation in time; for the benchmarks 28-40, CTD runs faster than HCTD, especially, for the benchmarks 38-40, HCTD is timeout and CTD solves the systems efficiently; for the benchmarks 41-49, both HCTD and CTD are timeout;

- for the benchmarks 14,31,32, 35 and 36 , the number of branches output by HCTD is much bigger than that output by CTD;

- for the benchmarks $6,10,12,29,30,32,35$ and 37 , the number of grapes output by HCTD is much bigger than that output by CTD. 


\section{Different Hierarchical Strategy}

To compute a $m$-HCTD for a given parametric system, as shown by Example 1 , we first take $\left\{x_{1}, \ldots, x_{n}\right\}$ as variable set and then add one parameter into the variable set at each recursive step. A different hierarchical strategy may be that we add a prescribed number ( first step and each recursive step.

The algorithm applying this different hierarchical strategy is called HCTDA and has been implemented as a function HCTDA. The comparing data of HCTD and HCTDA (for $s=1$ ) is shown in Table 3. It is indicated by Table 3 that

- for the benchmarks 3-11, HCTD runs faster than HCTDA, especially, for the benchmarks 10-11, HCTDA is timeout and HCTD completes the computation in time; for the benchmarks 12-18, HCTDA runs faster than HCTD, especially, for the benchmarks 19-20, HCTD is timeout and HCTDA completes the computation in time;

- the difference of the numbers of branches (grapes) output by HCTD and HCTDA is not striking.

In fact, we can input different $s$ when calling HCTDA. For many benchmarks in Table 2, the timings of different $s$ are similar. There are some benchmarks on which the timings of HCTDA differ greatly for different $s$. Due to page limitation, we do not report the timings here.

\section{Benefit of Hierarchical strategy}

We see that the benchmarks 41-49 in Table 2 are timeout when using both CTD and HCTD $(m=d)$. In fact, for some polynomial systems from practical areas, the complexity of computing comprehensive triangular decomposition is way beyond current computing capabilities. However for these systems (especially the systems with many parameters), we may try to compute the $m$-HCTD for $m=0, \ldots, d-1$. In this way, although we cannot solve the system completely, we may still get partial solutions.

We have tried the timeout benchmarks 41-49 in Table 2. The experimental results are shown in Table 4, where the columns " $m=0 "$, " $m=1 "$, " $m=2$ ", " $m=3$ " and " $m=4$ " denote the timings of calling Algorithm HCTD for $m=$ $0,1,2,3,4$; and the "error" mark means Maple returns an error message and stops computing. It is seen from the Table 4 that

- for all the benchmarks, we successfully get partial solutions;

- for most of the benchmarks, such as the benchmark 1 and benchmarks 3-7, we get results only when $m=0$.

Table 2 Comparing HCTD and CTD 


\begin{tabular}{|c|c|c|c|c|c|c|c|c|c|}
\hline & \multirow{2}{*}{ benchmark } & \multirow{2}{*}{$d \mid$} & \multirow{2}{*}{$n$} & \multicolumn{2}{|c|}{ time } & \multicolumn{2}{|c|}{ branch } & \multicolumn{2}{|c|}{ grape } \\
\hline & & & & HCTD & CTD & HCTD & CTD & HCTD & CTD \\
\hline 1. & MontesS2 & 1 & 3 & 0. & 0. & 1 & 1 & 1 & 1 \\
\hline 2. & MontesS4 & 2 & 2 & 0. & 0. & 1 & 1 & 1 & 1 \\
\hline 3. & F8 & 4 & 4 & 0.437 & 1.014 & 18 & 14 & 14 & 9 \\
\hline 4. & Hereman-2 & 1 & 7 & 0.093 & 0.468 & 2 & 2 & 10 & 6 \\
\hline 5. & MontesS3 & 1 & 2 & 0. & 0.031 & 3 & 2 & 2 & 2 \\
\hline 6. & MontesS5 & 4 & 4 & 0.078 & 0.187 & 6 & 8 & 13 & 6 \\
\hline 7. & MontesS6 & 2 & 2 & 0.015 & 0.047 & 4 & 3 & 5 & 4 \\
\hline 8. & MontesS7 & 1 & 3 & 0.046 & 0.156 & 4 & 4 & 6 & 8 \\
\hline 9. & MontesS8 & 2 & 2 & 0. & 0.094 & 2 & 2 & 2 & 2 \\
\hline 10. & MontesS12 & 2 & 6 & 0.593 & 7.925 & 5 & 5 & 61 & 27 \\
\hline 11. & MontesS13 & 3 & 2 & 0.078 & 0.265 & 6 & 9 & 9 & 8 \\
\hline 12. & MontesS14 & 1 & 4 & 0.452 & 4.353 & 6 & 3 & 28 & 12 \\
\hline 13. & MontesS15 & 4 & 8 & 0.187 & 0.889 & 5 & 5 & 14 & 12 \\
\hline 14. & MontesS16 & 31 & 12 & 1.198 & 1.825 & 37 & 8 & 11 & 7 \\
\hline 15. & Bronstein & 2 & 2 & 0.015 & 0.219 & 6 & 7 & 7 & 7 \\
\hline 16. & AlkashiSinus & 3 & 6 & 0.094 & 0.437 & 8 & 6 & 8 & 6 \\
\hline 17. & Lanconelli & 7 & 4 & 0.28 & 0.546 & 14 & 11 & 7 & 5 \\
\hline 18. & zhou1 & 3 & 4 & 0.047 & 0.156 & 5 & 5 & 5 & 5 \\
\hline 19. & zhou2 & 6 & 7 & 0.671 & 2.09 & 17 & 18 & 19 & 16 \\
\hline 20. & zhou6 & 3 & 3 & 0.031 & 0.218 & 6 & 4 & 6 & 5 \\
\hline 21. & SBCD13 & 1 & 3 & 0.015 & 0.094 & 2 & 2 & 9 & 6 \\
\hline 22. & SBCD23 & 1 & 3 & 0.202 & 0.344 & 4 & 2 & 15 & 12 \\
\hline 23. & $\mathrm{~F} 2$ & 2 & 2 & 0.032 & 0.234 & 3 & 3 & 3 & 3 \\
\hline 24. & F3 & 4 & 1 & 0.063 & 0.905 & 5 & 6 & 5 & 6 \\
\hline 25. & F5 & 3 & 2 & 0.046 & 0.11 & 6 & 3 & 3 & 3 \\
\hline 26. & F7 & 3 & 2 & 0. & 0.016 & 2 & 2 & 2 & 2 \\
\hline $2 \%$ & $\mathrm{~S} 2$ & 4 & 1 & 44.544 & timeout & 150 & & 92 & \\
\hline 28. & MontesS9 & 3 & 3 & 0.693 & 0.468 & 21 & 13 & 16 & 13 \\
\hline 29. & MontesS10 & 3 & 4 & 0.421 & 0.359 & 13 & 6 & 19 & 6 \\
\hline 30. & MontesS11 & 3 & 3 & 0.858 & 0.655 & 12 & 16 & 20 & 10 \\
\hline 31. & F4 & 4 & 2 & 11.637 & 0.375 & 20 & 3 & 3 & 3 \\
\hline 32. & zhou 5 & 4 & 5 & 5.616 & 2.902 & 51 & 19 & 97 & 22 \\
\hline 33. & F6 & 4 & 1 & 0.296 & 0.14 & 13 & 3 & 11 & 3 \\
\hline 34. & MontesS1 & 2 & 2 & 0.016 & 0. & 4 & 2 & 3 & 3 \\
\hline 35. & Hereman-8-8 & 3 & 5 & 96.439 & 10.468 & 108 & 9 & 161 & 14 \\
\hline 36. & S3 & 4 & 3 & 2.618 & 1.436 & 35 & 13 & 17 & 11 \\
\hline $3 \%$ & Maclane & 3 & 7 & 5.242 & 4.009 & 17 & 9 & 155 & 27 \\
\hline 38. & S1 & 3 & $2 \mid t$ & timeout & 4.04 & & 10 & & 10 \\
\hline 39. & Neural & 1 & $3 t$ & timeout & 0.188 & & 2 & & 7 \\
\hline 40. & Gerdt & 3 & $4 t$ & timeout & 0.842 & & 4 & & 6 \\
\hline 41. & Lazard-ascm2001 & 3 & $4 t$ & timeout & timeout & & & & \\
\hline 42. & Leykin-1 & 4 & $4 t$ & timeout & timeout & & & & \\
\hline 43. & Cheaters-homotopy-easy & 4 & $3 t$ & timeout & timeout & & & & \\
\hline 44. & Cheaters-homotopy-hard & 5 & $2 t$ & timeout & timeout & & & & \\
\hline 45. & Lazard-ascm2001 & 3 & $4 t$ & timeout & timeout & & & & \\
\hline 46. & MontesS18 & 2 & $3 t$ & timeout & timeout & & & & \\
\hline 47 & Pavelle & 4 & $4 t$ & timeout & timeout & & & & \\
\hline 48. & p3p & 5 & $2 t$ & timeout & timeout & & & & \\
\hline 49. & z3 & 6 & $11 \mathrm{t}$ & timeout & timeout & & & & \\
\hline
\end{tabular}


Table 3 Comparing HCTD and HCTDA (for $s=1$ )

\begin{tabular}{|c|c|c|c|c|c|c|c|c|c|}
\hline & \multirow{2}{*}{ benchmark } & \multirow{2}{*}{$d$} & \multirow{2}{*}{$n$} & \multicolumn{2}{|c|}{ time } & \multicolumn{2}{|c|}{ branch } & \multicolumn{2}{|c|}{ grape } \\
\hline & & & & HCTD & HCTDA & HCTD & HCTDA & HCTD & HCTDA \\
\hline 1. & MontesS5 & 4 & 4 & 0.078 & 0.078 & 6 & 6 & 13 & 13 \\
\hline 2. & zhou1 & 3 & 4 & 0.047 & 0.047 & 5 & 5 & 5 & 6 \\
\hline 3. & MontesS9 & 3 & 3 & 0.693 & 0.796 & 21 & 21 & 16 & 27 \\
\hline 4. & Monte & 3 & 3 & 0.858 & 1.207 & 12 & 24 & 20 & 38 \\
\hline 5. & Monte & 2 & 6 & 0.593 & 0.671 & 5 & 5 & 61 & 60 \\
\hline 6 & AlkashiSinus & 3 & 6 & 0.094 & 0.109 & 8 & 10 & 8 & 10 \\
\hline 7. & tein & 2 & 2 & 0.015 & 0.031 & 6 & 5 & 7 & 6 \\
\hline 8. & & 2 & 2 & 0.046 & 0.266 & 4 & 6 & 4 & 6 \\
\hline 9. & SBCD13 & 1 & 3 & 0.015 & 0.031 & 2 & 2 & 9 & 7 \\
\hline 10. & & 4 & 1 & 0.296 & timeout & 13 & & 11 & \\
\hline 11. & $\mathrm{~S} 2$ & 4 & 1 & 44.544 & timeout & 150 & & 92 & \\
\hline 12. & Maclar & 3 & 7 & 5.242 & 2.605 & 17 & 13 & 155 & 122 \\
\hline 13. & SBCD23 & 1 & 3 & 0.202 & 0.109 & 4 & 2 & 15 & 13 \\
\hline 14. & & 4 & 2 & 11.637 & 1.653 & 20 & 26 & 3 & 3 \\
\hline 15. & MontesS15 & 4 & 8 & 0.187 & 0.124 & 5 & 5 & 14 & 14 \\
\hline 16. & F8 & 4 & 4 & 0.437 & 0.358 & 18 & 16 & 14 & 11 \\
\hline 17. & MontesS16 & 3 & 12 & 1.198 & 0.951 & 37 & 21 & 11 & 8 \\
\hline 18. & & 4 & 3 & 2. 618 & 1.81 & 35 & 29 & 17 & 15 \\
\hline 19. & Neural & 1 & 3 & timeout & 0.296 & & 6 & & 15 \\
\hline 20. & Gerdt & 3 & 4 & timeout & 288.352 & & 4 & & 11 \\
\hline
\end{tabular}

Table 4 Timings of $m$-HCTD for different $m$

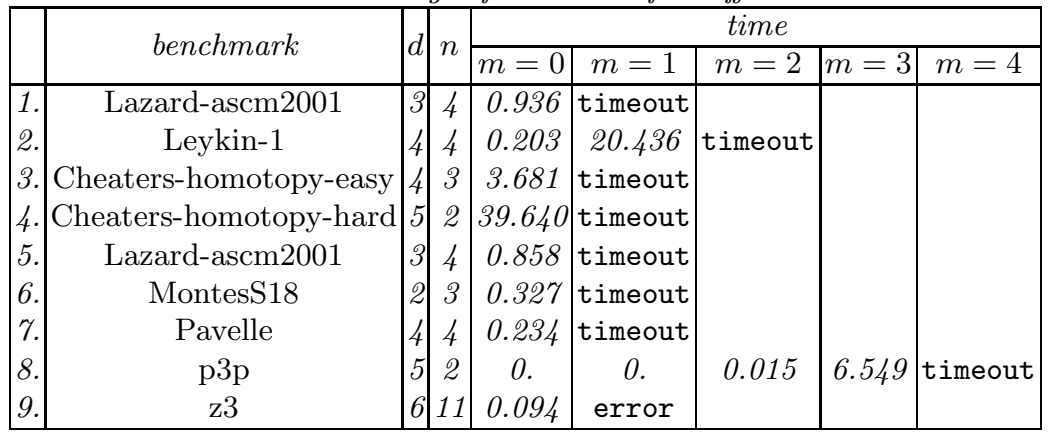

\section{References}

1. P. Alvandi, C. Chen, M. Moreno Maza: Computing the limit points of the quasicomponent of a regular chain in dimension one. Computer Algebra in Scientific Computing, 30-45, 2013.

2. P. Aubry, D. Lazard, M. Moreno Maza: On the theories of triangular sets. J. Symb. Comp., 28: 105-124, 1999.

3. C. Chen, J. Davenport, J. P. May, M. Moreno Maza, B. Xia, R. Xiao: Triangular decomposition of semi-algebraic systems. Proc. ISSAC, 187-194, 2010.

4. C. Chen, J. Davenport, M. Moreno Maza, B. Xia, R. Xiao: Computing with semialgebraic sets represented by triangular decomposition. Proc. ISSAC, 75-82, 2011.

5. C. Chen, O. Golubitsky, F. Lemaire, M. Moreno Maza, W. Pan: Comprehensive triangular decomposition. Proc. CASC 2007, LNCS 4770, 73-101, 2007. 
6. C. Chen, M. Moreno Maza: Algorithms for computing triangular decomposition of polynomial systems. J. Symb. Comp., 47 (6): 610-642, 2012

7. S.-C. Chou: Mechanical geometry theorem proving. Springer, 1988.

8. Z. Chen, X. Tang, B. Xia: Generic regular decompositions for parametric polynomial systems. Accepted by Journal of Systems Science and Complexity, 2013. arXiv:1301.3991v1.

9. X.-S. Gao, S.-C. Chou: Solving parametric algebraic systems. Proc. ISSAC, 335341, 1992

10. X.-S. Gao, X. Hou, J. Tang, H. Chen: Complete solution classification for the perspective-three-point problem. Pattern Analysis and Machine Intelligence, IEEE Transactions on, 25 (8), 930-943, 2003.

11. M. Kalkbrener: A generalized euclidean algorithm for computing for computing triangular representationa of algebraic varieties. J. Symb. Comp., 15: 143-167, 1993.

12. D. Kapur, Y. Sun, D. Wang: A new algorithm for computing comprehensive gröbner systems. Proc. ISSAC, 25-28, 2010.

13. M. Moreno Maza: On triangular decompositions of algebraic varieties. Technical Report TR 4/99, NAG Ltd, Oxford, UK, 1999.

14. A. Montes, T. Recio: Automatic discovery of geometry theorems using minimal canonical comprehensive Gröbner systems. ADG 2006, LNAI 4869, 113-138, 2007.

15. K. Nabeshima: A speed-up of the algorithm for computing comprehensive gröbner systems. Proc. ISSAC, 299-306, 2007.

16. A. Suzuki, Y. Sato: An alternative approach to comprehensive gröbner bases. Proc. ISSAC, 255-261, 2002.

17. A. Suzuki, Y. Sato: A simple algorithm to compute comprehensive gröbner bases. Proc. ISSAC, 326-331, 2006.

18. X. Tang, Z. Chen, B. Xia: Generic regular decompositions for generic zerodimensional systems. Accepted by Science China: Information Sciences, 2012. DOI: 10.1007/s11432-013-5057-5.

19. D. K. Wang: Zero decomposition algorithms for system of polynomial equations. Computer Mathematics, World Scientific, 67-70, 2000.

20. D. M. Wang: Computing triangular systems and regular systems. J. Symb. Comp., 30: 221-236, 2000.

21. D. M. Wang: Elimination methods. Springer, 2001.

22. D. M. Wang: Elimination practice: software yools and applications. Imperial College Press, 2004.

23. V. Weispfenning: Comprehensive gröbner bases. J. Symb. Comp., 14: 1-29, 1992.

24. W.-T. Wu: Basic principles of mechanical theorem proving in elementary geometries (in Chinese). Science in China Series A Mathematics, 507-516, 1977.

25. L. Yang, X. Hou, B. Xia: A complete algorithm for automated discovering of a class of inequality-type theorems. Science in China Series $\mathbf{F}$ Information Sciences, 44 (1): 33-49, 2001.

26. L. Yang, B. Xia: Automatic inequality proving and discovering (in Chinese). Science Press, 2008.

27. L. Yang, J. Zhang: Searching dependency between algebraic equations: An algorithm applied to automated reasoning. International Centre for Theoretical Physics, 1-12, 1990.

28. L. Yang, J. Zhang, X. Hou: Non-linear algebraic formulae and theorem automated proving (in Chinese). Shanghai Education Technology Publishers, 1992. 